Presented at the Conference on

Instrumentation for Heavy Ion Nuclear

Research, ORNL, Oaik Ridge, TN, October

LBL--18642

$22-24,1984$

THE BERKELEY HIGH-RESOLUTION BALL*

R.M. DIAMOND

Nuclear Science Divis ion

Lawrence Berkeley Laboratory

University of California

Berkeley, California 94720

For the Conference on Instrumentation for Heavy Ion

Nuclear Reseaich, Oak Ridge National Laboratory

\title{
DISCLAIMER
}

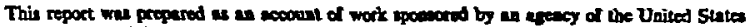
Governmeat. Neither the Unitad States Gomernmeat nor any ancacy threof, nor eny of their

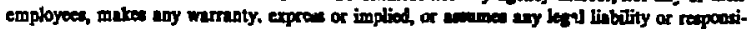

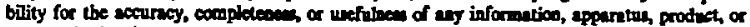

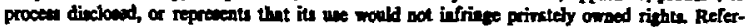

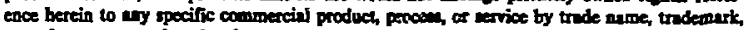

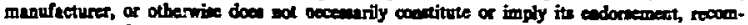

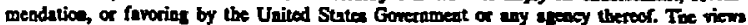
and opinions of autho, expreaned bersin do bot secenerily atte or refect thowe of the United States Governmest or any arency thereat.

*Th is work was supported by the Director, Off ice of Energy Research, Division of Nuclear Physics of the Off ice of High Energy and Nuclear Physics of the U.S. Department of Energy under Contract DE-ACO3-76SF00098. 


\title{
THE BERKELEY HIGH-RESOLUTION BALL*
}

\author{
R.M. DIAMOND \\ Nuclear Science Division \\ Lawrence Berkeley Laboratory \\ University of California \\ Berkeley, California 94720
}

Criteria for a high-resolution r-ray system are discussed. Desirable properties are high resolution, good response function, and moderate sol id angle so as to ach:eve not only double- but triplecoincidences with good statistics. The Berkeley High-Resolution Bail Involved the first use of bismuth germanate (BGO) for anti-Compton shields for be detectors. The resulting compact shield permitted rather close packing of 21 detectors around a target. In addition, a small central BGO ball gives the total r-ray energy and multiplicity. as weil as the angular pattern of the $r$ rays. The 21-detector array is nearly complete, and the central ball has been designed, but not yet constructed. First results taken with 9 detector modules are shown for the nucleus $156 \mathrm{Er}$. The complex decay scheme indicates a transition from collective rotation (prolate shape) to single-particle states (possibly oblate) near spin $30 \mathrm{~h}$, and has other interesting features.

*This work was supported by the Director, Office of Energy Research, Division of Nuclear Physics of the Office of $H$ igh Energy and Nuclear Physics of the U.S. Department of Energy under Contract DE-ACO3-76SF00098. 


\section{R.M. DIAMOND}

Four or more years ago frali: Stephens and I becane interested in developing a high-resolution r-ray system, both to extend discrete-line spectroscopy and to study continuum r-rays. If it were possible to push discrete transition work 10-15 spin units higher, all the detafled studies possible at iower spins could be carried out and much more could be learned about how nuclef carry angular momentum and how they change with spin. Questions to be answered are how rapidly do the pairing correlations quench, do alignments and backbends continue to high spin, and what are the changes in shape and in collective motion with increase in spin? To achieve this goal appeared to require both the "looking dow" technique of the Nal balls then being constructed, namely, to select a limited population of initial states by cuts on the fold distribution and on the total-energy response of the ball, and a looking up" scheme in which a gate is set on a discrete line of moderately high spin and the transitions above it are looked for in coincidence. But the highest spin lines obseried might represent at most a few percent of the total population at that spin, and to learn about the majority of the states and their properties, one would also have to study the continuum spectrum. He had already observed, by comparing Nal and Ge $r-r$ currelation spectra, that there were structures in the continuum region that required 5-10 $\mathrm{KeV}$ resolution to be seen. This is better than can de done, with NaI detectors, but, as explained below, a good response function is also very important in coincidence studies and Ge detectors are poor in that regard.

So at that time we were able to convince $C$. Gruhn to undertake a feasibility study of liquid Xe detectors as

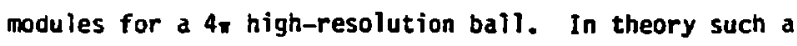


systen would have an intrinsic resolution only a factor of two worse than solid-state Ge cetectors, and it has a number of attrac sponse function. But this undertaking proved to be an ongoing developmental problem, principally because of very low-level (ppb) impurities, and appeared that it wight take years to bring to fruition. So after a year we decided to base our multidetector system on already existing Ge detectors. However, you have already heard at this Conference from $\mathrm{Dr}$. :-indblad ${ }^{\mathrm{l}}$ on the progress they have made in Stockholm during the last three years with liquid $\mathrm{Ar}$ and $\mathrm{Xe}$ counters, including the operation of a liquid xe detector with about $8 \mathrm{KeV}$ resolution for $-1 \mathrm{MeV} r$ rays. So such counters still may live up to their theoretical promise.

TABLE I Criteria for optimum r-ray system.

1. High energy resolution

2. Good response function

3. Good efficiency

4. Total-energy spectrometer

5. Multiplicity filter

6. Prompt initial timing signal

In Table I are listed three primary criteria important for a high-resolution array, and three secondary features we wanted. As mentioned, Ge detectors provide the highest resolutioi possible today with reasonable efficiency for $\gamma$-ray energies in the range of $50 \mathrm{KeV}$ to a few $\mathrm{MeV}(-2 \mathrm{KeV}$ resolution at $1 \mathrm{MeV})$. Can we do better? Hith a single detector, no. In this case the average resolution can be defined as the reciprocal of the number of resolvable points in the energy range of interest. Consider a range 

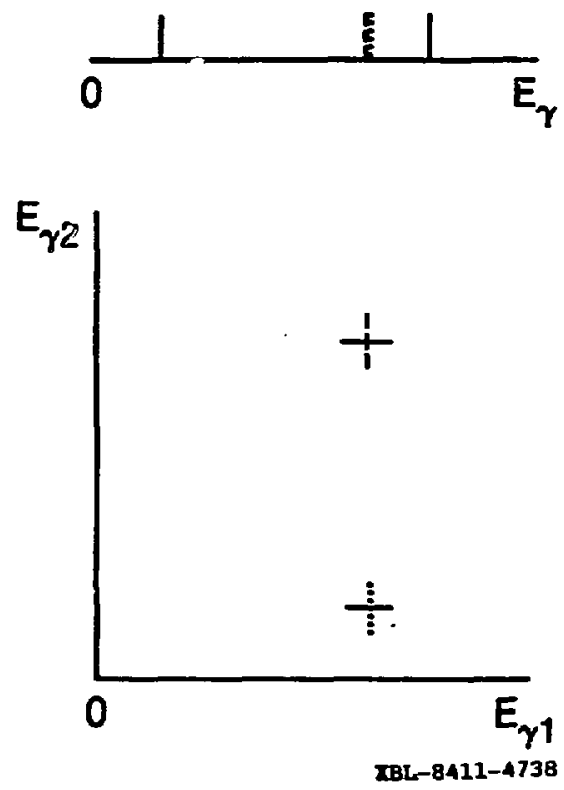

FIGURE 1 The over lapping lines in the singles spectrum, is are resolvable in the doubles spectrum by their different cascade partners.

of 0-1 MeV and a constant resolution of $2 \mathrm{KeV}$; this gives 500 resolvable points. If two $r$ rays fall on the same point, they cannot be resolved. But the $r$ rays we are interested in are usually not single events but members of a cascade and are correlated with the other members. If two (or more) detectors are used, the coincidence relationships between the two overlapping $r$ rays and the other members of their individual cascades may serve to resolve than, as illustrated in Fig. 1. The 500 resolvable points 


\section{THE BERKELEY HIGH-RESOLUTION BALL}

of the single detector have become $500^{2}$ points in the two-dimensional array of the two coincident detectors, and it is much iess probable that the two pairs of $r$ rays coincide than the original single lines. Stfll higher order coincidences give still higher effective resolution for coincident cascades of $r$ rays by enormousiy increasing the number of possible resolvable points; a triple coincidence would have $500^{3}$ possible resolvable points. Thus, a desirable feature for our array is to have as many detectors as close as is reasonably possible (see below) to the target, in order to favor high-order coincidences.

A second criterion is to have a good response function, f.e., every $y$ ray that strikes the detector should result in a voltage pulse proportional to the original r-ray energy. A Ge counter does not fulfill this requirement very well. It is a relatively low 2 , moderately dense materlal that interacts with $\gamma$ rays in the energy range of interest principally by Compton scattering. With a $5 \times 5$ $\mathrm{cm}$ Ge detector (approximately $20 \%$ the efficiency of a $7.6 \times$ $7.6 \mathrm{~cm} \mathrm{NaI}$ detector for $1.33 \mathrm{MeV}$ r rays) a $1.33 \mathrm{MeV}$ r ray has an absorption probabllity of $-3 / 4$ and a peak to total $(P / T)$. ratio of $15-20 \%$. This means that $-1 / 4$ of such $\gamma$ rays do not interact at all with the detector, and that of those that do, only 15-20\% give useful full-energy peaks. In a doubles coincidence measurement, only $2-4 \%$ of the events obtained are good peak-peak values; the remaining 96-98\% are unwanted garbage. This is a very undesirable situation, and the solution has been known for some time; put Compton-suppression shields around the Ge detectors. The preceding talk by Dr. Twin on TESSA illustrated how this was done at Daresbury ${ }^{2}$; large $20 \times 25 \mathrm{~cm}$ Nal shields were placed on six Ge detectors arranged around the target, 


\section{R.M. DIAMONO}

giving a markedly improved peak/total ratio and resulting in the beautiful spectra you have just seen. Mot having such NaI shields on hand, we decided to try for bismuth germanate (BGO) shields, for, if successful, they offered a great advantage. The material is considerably denser than Nal $\left(7.13\right.$ to $\left.3.67 \mathrm{~g} / \mathrm{cm}^{3}\right)$ and of higher averge $Z$, so that it has a y-ray absorption length 2-1/2 times smaller than Nal. This means more compact shields, so that more Comptonsuppressed detectors can be placed near the target to give the higher order coincidences discussed in the previous paragraph. But bismuth germanate also has a serious handicap; the light output is only 10-15\% that of Nal. In addition, at that time (1981) the companies that marketed it had not made BGO crystals large enough to serve this purpose, as shields of the order of $13 \mathrm{~cm}$ or more in diameter and length were needed.

It took a year to obtain a prototype yersion of the BGO shield. The unit was not quite large enough, and it had broken in two during machining and had been cemented back together. Indeed it looked as if it might be a long, hard period before single crystals of $B 60$ of sufficient size could be processed into suitable shields. But upon testing the broken protatype, the cemented fracture did not seem to cause any problem, and the taper at the front of the shield appeared to reflect light so well that the phototubes at the back gave a bigger signal when the source was placed on the front piece (whose light had to pass through the fracture) than when it was put on the back piece. So the problem was solved by making the shield in six longitudina? pieces that were put together to form the detector shell of suitable size. I believe that all of the Compton suppression shields that have been made from $B 60$ 
THE BERKELEY HIGH-RESOLUTIOH BALL

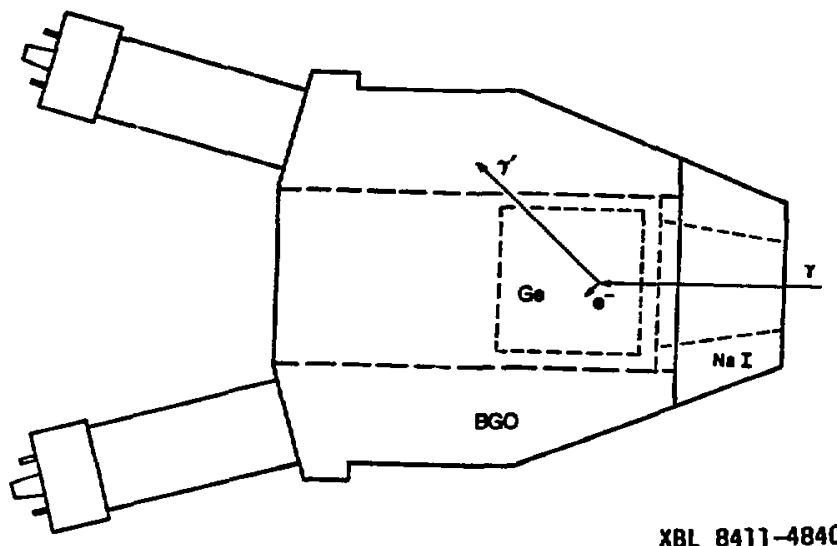

FIGURE 2 Out line of $B G 0$ Compton-suppression shield showing $5 \times 5 \mathrm{~cm}$ Ge detector inside. two of six phctomultiplier tubes on back surface, and MaI "cap" on front taper.

have been produced in this way. In our design a 1-1/2" photomultiplier tube was placed on the back of each of the six sections to gather the light as efficiently as possible. A schematic drawing of our BGO shield showing two of the tubes is given in Fig. 2. It is a coaxial design, so that there is no shield material at the front opening where the $\gamma$ rays come in to the Ge detector, nor at the rear where the snout of the Ge detector enters. Lack of coverage at the latter position means that Compton-scattered $r$ rays in the angular range of $0^{\circ}-25^{\circ}$ (multiple compton scattering in the Ge crystal and primary interactions throughout the whole volume of the Ge smear these angles out) will not be caught by the shield, leaving the complementary non-full-energy transition in the resulting 


\section{R.M. DIAMOND}

spectrum. He do not believe these low-energy transitions puse a serious problem, although there are attempts being made to reduce its magnitude by reducing the snout behind the Ge detector to a minimum diameter and then surrounding it with additional shield material. (Of course the transverse shields do not have this hole, but they have one at the side instead.) But the opening at the front is more disturbing, as the (low energy) $r$ rays Compton-scattered through about $135^{\circ}-180^{\circ}$ correspond to leaving in the spectrum the Compton edges just below the full energy peaks. The edges appear just where we are interested in having a clean, low-background region. Figure 3 shows two spectra of ${ }^{60}$ Co taken with one of our Ge detectors, a Comptonsuppressed one taken with the BGO snield and an unsuppressed one normalized to the same full-energy peak heights. (In the former, the Compton-edge peaks just mentioned can be clearly seen.) The peak/total ratio for ${ }^{60}$ Co above a $300 \mathrm{KeV}$ threshold improves fran $-20 \%$ to $50 \%$. But for coincidence experiments there is the even more marked improvement shown in Table II. Note, for example, that with bare be detectors full-energy triple TABLE II Ge detector $20 \%$ (counts above $300 \mathrm{KeV}$ ).

\begin{tabular}{lcccc}
\hline & Gel & Ge 2 & $G^{3}$ & $G^{4}$ \\
\hline Peak/Total & 0.2 & 0.04 & 0.008 & 0.0016 \\
Compton-suppressed & 0.5 & 0.25 & 0.13 & $0.06-$ \\
Peak/Tota? & & 6 & 16 & 39 \\
\hline Improvement Factor & 2.5 & 6 & 16 & \\
\hline
\end{tabular}

coincidences are only $0.8 \%$ of the events, so that triple coincidences have not been used in in-beam spectroscopy. But with a $50 \% \mathrm{P} / \mathrm{T}$ ratio, $12.5 \%$ are good events making them 


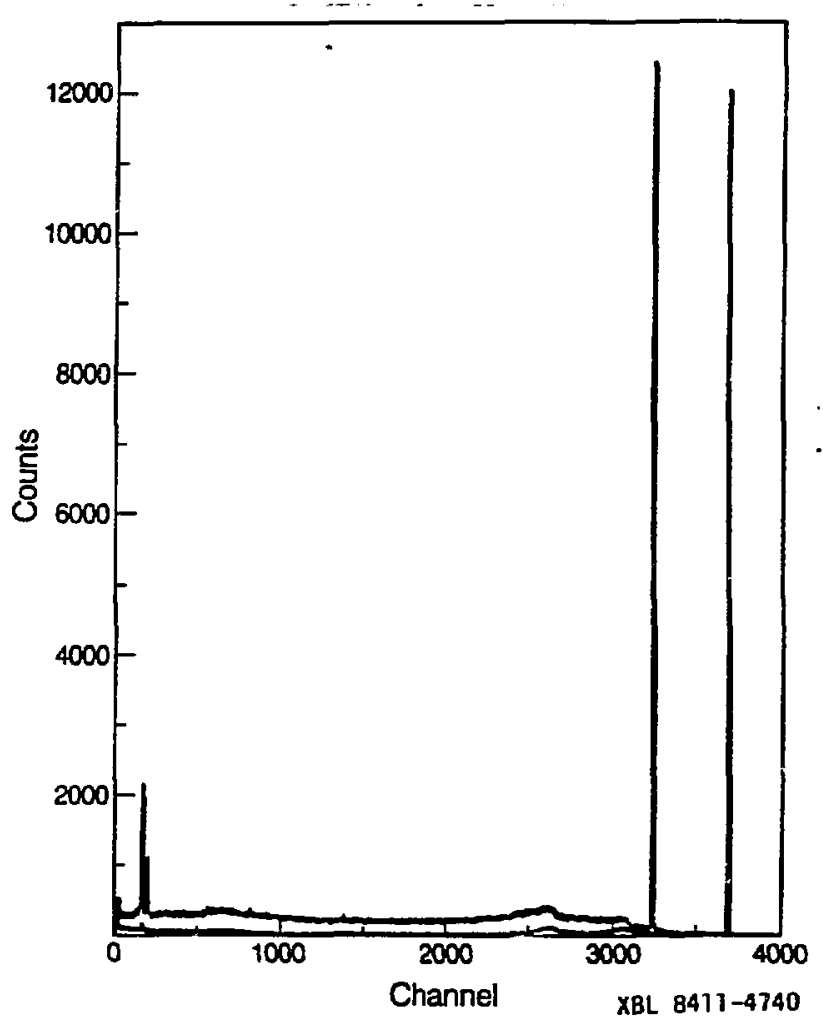

FIGURE 3 Spectra of 60 co taken with bare $20 \%$ Ge detec- tor and with Compton-suppressed detector (no NaI cap).

quite useable. And still higher ratios are possible. By the addition of a MaI "cap" to the front of the BGO shield, as shown schematically in Fig. 2, we have obtained a $P / T$ ratio of $55 \%$ for the ${ }^{60}$ Co peaks (300 KeV threshold) and, 
most importantly, have almost completely wiped out the Compton-edge peaks. Other designs using larger $B G 0$ shields can give P/T ratios of $60-65 \%$ for ${ }^{60} \mathrm{Co}$, so tremendous improvenent in the Ge response function is possible.

The system efficiency involves the questions of how many Ge detectors at what distance from the target. With the design shown in Fig. 2 involving $13.5 \times 13.5 \mathrm{~cm}$ shields, some units will touch at their tapered cones when 13-14 $\mathrm{cm}$ from the target. If we take $15 \mathrm{~cm}$ as the distance from the target to the face of the $5 \times 5 \mathrm{~cm}$ Ge detector inside the BGO shield, we find we can place 21 detectors at this distance and still leave the top and botton above the target clear. This will be necessary to accomodate the photomultiplier tubes for a small, central BGO ball between the target and the Ge modules. Of course, the number of detectors is a matter of choice and depends somewhat on the geometrical arrangement selected. Too small a number hurts the higher order coincidences ard the statistics; too large a number is costly, and after a moderately large number one does not gain very rapidly, as additional detectors require an expansion in the radial distance from the target. Another consideration on the distance from the target involves the amount of suming to be tolerated in an individual detector. At $15 \mathrm{~cm}$ from the target, the geometric solid angle of our detectors, diminished by tire Ge transparency, is $-5 \times 10^{-3}$. Sixty percent (or more) of such, rays interacting with the ge detector are rejected by the shield (plus Nal cap) so the overall efficiency is $\leq 2 \times 10^{-3}$. If the r-ray cascades have an average multiplicity of 20 , this gives $-4 \%$ suming, on average. This is probably $0 . K$. for mast purposes; if not the geometric efficiency must be reduced. 
THE BERKELEY HIGH-RESOLUTION BAL

Lastiy, but not least, Doppler effects wust be considered. The usual method of producing muclei in high-spin states is with ( $H .1 ., \times n)$ reactions. In a typical case, $-180 \mathrm{Mey}{ }^{40} \mathrm{Ar}$ on ${ }^{124} \mathrm{Sn}$, the recofling compound mucleus nas a velacity $-2.5 \%$ that of 11ght. The Doppler shift for a $y$ ray emitted by such a moving source is

$$
\frac{E-\varepsilon_{0}}{E_{0}}=\frac{y}{c} \cos \theta
$$

where $\theta$ is the angle between the detector and the recoiling nucleus. So near $0^{\circ}$ or $180^{\circ}$ the Doppler shift is quite large, $-25 \mathrm{KeV}$ for a $1 \mathrm{MeV}$ y ray. But the broadening due to the finite opening-half-angle of the detector

$$
\frac{\Delta E}{E_{0}} \approx 2 \frac{y}{C} \sin \theta \sin
$$

is quite $5 m a l 1, \leq 1 / 2 \mathrm{KeV}$ for o near $0^{\circ}$ or $180^{\circ}$ and $=$ $9.5^{\circ}$. By using a thin target or multiple thin targets, the full Drppler shift is obtained, but since this corresponds to a change in gain it can be corrected for in the axplifier or $A D C$ or in the analysis progran with no loss in resolution. On the other hand, for angles of o near $90^{\circ}$, the Doppler shift becones small, but the broadening becomes a maximum. For the $180 \mathrm{MeV}{ }^{40} \mathrm{Ar}+{ }^{124} \mathrm{Sn}$ example, $\Delta E / E_{0} \approx 0.8 \%$ or $8 \mathrm{KeV}$ broadening for a 1 MeV transition. Such a smearing of the energy ruins the resolution of the detector, but can be reduced or avoided altrgether under certain conditions. For example, if such a product nucleus recoils out of the target into a lead or gold backing, it will stop in the order of picoseconds. If the transitions are emitted after stopping, there will be no Doppler shift or broadening. Thus, nuclei that do not have fast, col- 


\section{R.M. DIAMONO}

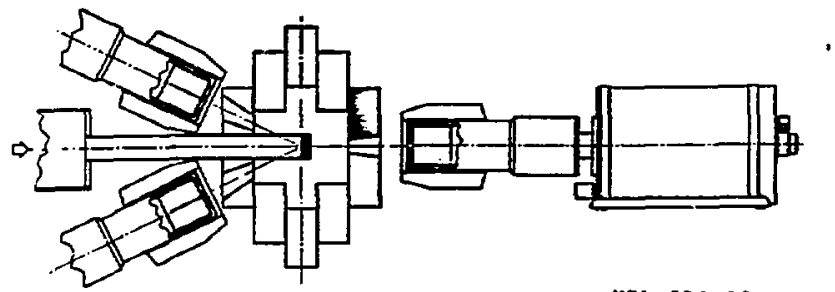

XBL 819-2052

FIGURE 4 Vertical cut through the High-Resolution 8 all system, showíng one $G \in$ detector in each ring. Not shown are photomultiplier tubes and NaI caps on $\mathbf{8 6 0}$ shields.

lective transitions in their de-excitation cascades can, and do, take picoseconds to de-excite and da not present a problem. But if the $r$ rays are fast, something has to be done to alleviate the Doppler broadening; the detectors must be pulled back further or collimated or both in order to decrease the detector opening angle, and thus the broadening.

With these considerations in mind we have designed 3 the High-Resolution Ball to consist of 21 BGD-shielded Ge detectors arranged (in three rings of seven detectors) around a smal?, central "ball" or "castle" of 40 BGO sectors. Some of the latter have holes through which the Ge detectors see the target, and the $\mathbf{4 0}$ together form a sum spectrometer and multiplicity filter around the target, as well as giving the angular pattern of the $r$ rays emitted. A sideview of the arrangement is given in Fig. 4, which shows one detector from each ring. Not shown are the NaI caps on the BGO shields, nor are the photomultiplier tubes drawn in on either the shields or the central ball sectors. For the BGO sectors in the upper half of the central 


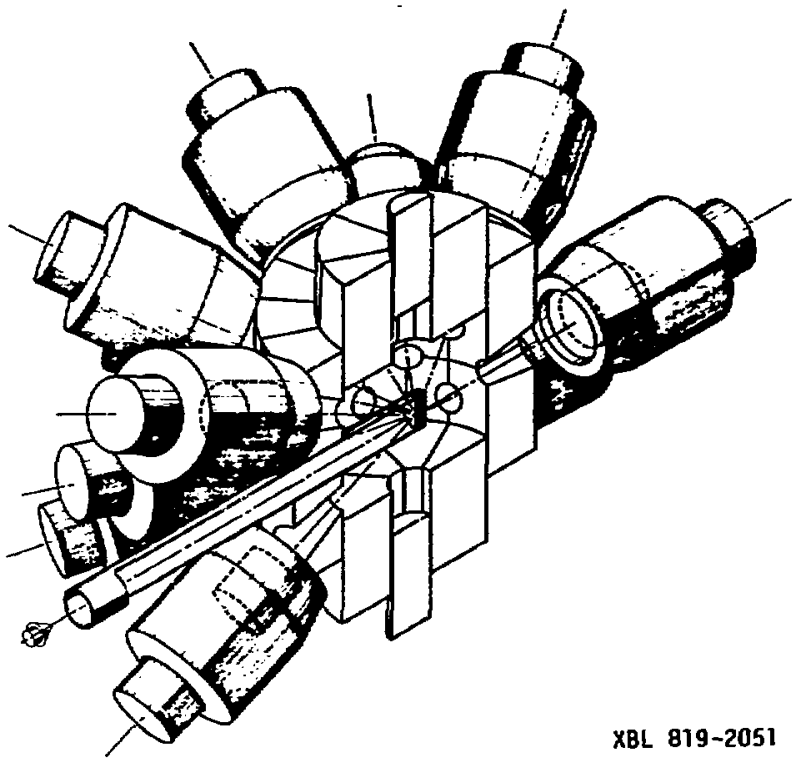

FIGURE 5 Perspective view of $-1 / 2$ the system.

ball, the photomultiplier tubes go up from the top faces of the sectors, and for those in the lower half, the tubes go downwards from the bottom faces. A cut-away perspective drawing of the system is shown in Fig. 5.

With 21 of our detectors at $15 \mathrm{~cm}$ from the target, an event rate of $10^{5}$ /second (the order of our usual rate in a $\left({ }^{40} \mathrm{Ar}, \mathrm{xn}\right)$ reaction), and an average $r$-ray multiplicity of 20 , we estimate the double-, triple-, and quadruplecoincidence rates to be $11 \mathrm{k} / \mathrm{s}, 2.2 \mathrm{k} / \mathrm{s}$ and $280 / \mathrm{s}$, respectively. We have already performed experiments with 9, 12, and 15 Compton-suppressed Ge detectors (but without the 


\section{R.M. DIAMOND}

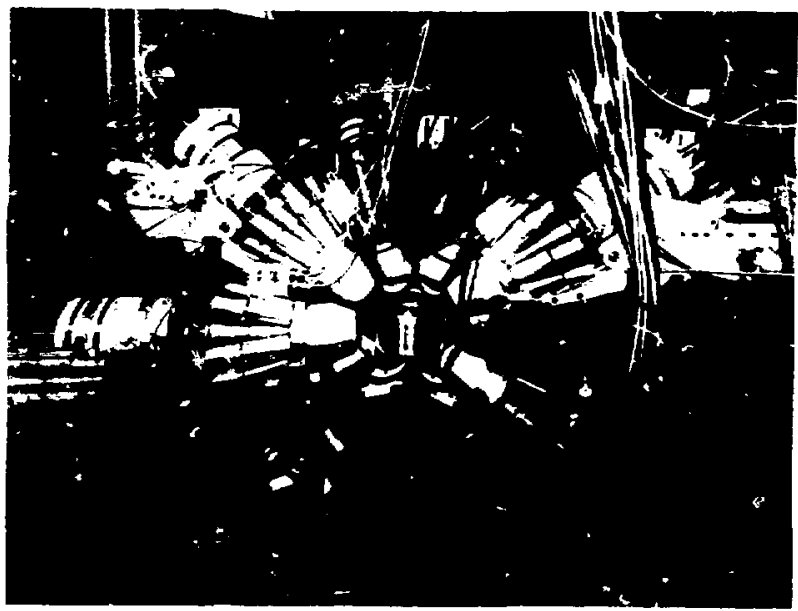

CEB $846-4587$

FIGURE 6 Photograph of 12 Compton-suppressed Ge modules in place aro:end a small target chamjer.

central BGO ball), and do get the corresponding calculated rates.

Where we were 3-4 months ago (Sumer, 1984) is shown in Fig. 6, a photograph of 12 Compton-suppressed Ge modules in place around the target inside a small chamber. The beam-line to the chamber passes between the two middle modules in the horizontal plane. The Ge detectors are out of sight inside the $B G O$ shields, but their small liquidnitrogen dewars are visible, as are the pre-amps for the shields. He now have all $21 \mathrm{Ge}$ detectors and 16 of the BGO shields; the last of the latter are due by February, 1985. We shall go out to bid for the NaI caps for the BGO shields before the end of this year. By the beginning of 1985 we 
should have a new systew of fast B-channel multiplexer's and of ADC's and a fast CAMAC crate in operation for acquiring data. All that will rexain to build is the central BCiO ball, and its design has been completed.

One of the goals of the High-Resolution Ball is to push the limits of discrete $r$-ray spectropscopy to higher spin into the bottom of the present continuum region, in orcer to gain the much more detailed information possible with discrete-line studies. This will be accomplished by cutting down the number of de-excitation pathways being observed both by cuts on the total energy and multiplisity measured with the central ball, and by simultaneously gating on single, high-spin discrete lines (and "looking up" in coincidence above the last kncwn discrete lines). Since we do not have a central ball yet, we can only do the latter at present, and for our first experiments we have chosen to look at the region around $Z=64$ and $N \geq 82$. We have taken data on some eleven nuclei. This choice wa:s made for three reasons. First of all, most nuclei in this region are expected to have long enough feeding times (picoseconds) into the range of high-spin states wich we want to study that they will be stopped in lead-backed targets before they emit their de-excitation r rays. Thu:i, there will be no problem with Doppler broadening. Secondly, the level schemes are apt to be quite complex, as these are not good collective rotors having bands with approxi- mately rotational spacings, but involve mainly singleparticle states with possibly weakly collective interactions. For this reason many of then have not yet been studied to high spin and they represent a challenge, though a difficult one. Thirdly, they are in a region where the physics appears to be varied and interesting, involving 


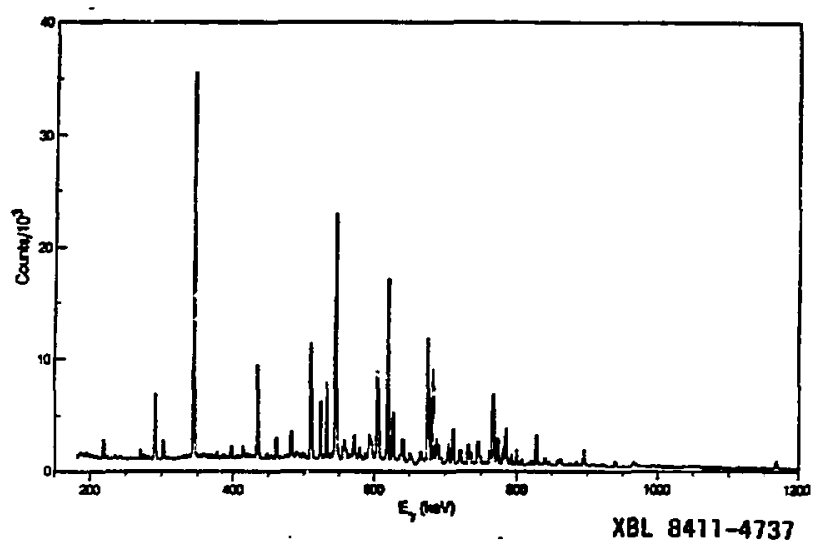

FIGURE 7 Spectrum of ${ }^{156} \mathrm{Er}$ in coincidence with the 452 $\mathrm{KeV}, 4^{+} \rightarrow 2^{+}$ground-band transition.

shape changes, and possibly the theoretically predicted transformation to superdeformed shapes (2:1 axis ratio) at relatively low spins and also band terminations.

I shall show you some data on ${ }^{156} \mathrm{Er}$ which are being analyzed by Frank Stephens. They were taken with 9 Compton-suppressed Ge detectors in two days running at the 88" cyclotron with a -1 pna beam of $175 \mathrm{MeV}{ }^{40} \mathrm{Ar}^{8+}$ on a lead-backed $-1 \mathrm{mg} / \mathrm{cm}^{2}{ }^{120} \mathrm{Sn}$ target. The data consist of $-1.5 \times 10^{8}$ doutle- and $-10^{7}$ triple-soincidence events. A spectrun in coincidence with the $452 \mathrm{KeV}, 4^{+} \rightarrow 2^{+}$transition of the ground band shows the number of lines observed in ${ }^{156} \mathrm{Er}$, essentially clean of ${ }^{157,159} \mathrm{Er}$ (Fig. 7). Actual$l y$, the total $Y-Y$ projection spectrum is clean enough so that the small peak at $884 \mathrm{KeV}$, the $28^{+} \rightarrow 26^{+}$transition that has only $-3 \%$ the strength of the ground-band $4^{+} \rightarrow 2^{+}$ transition, can be used as a gate to provide the very nice 


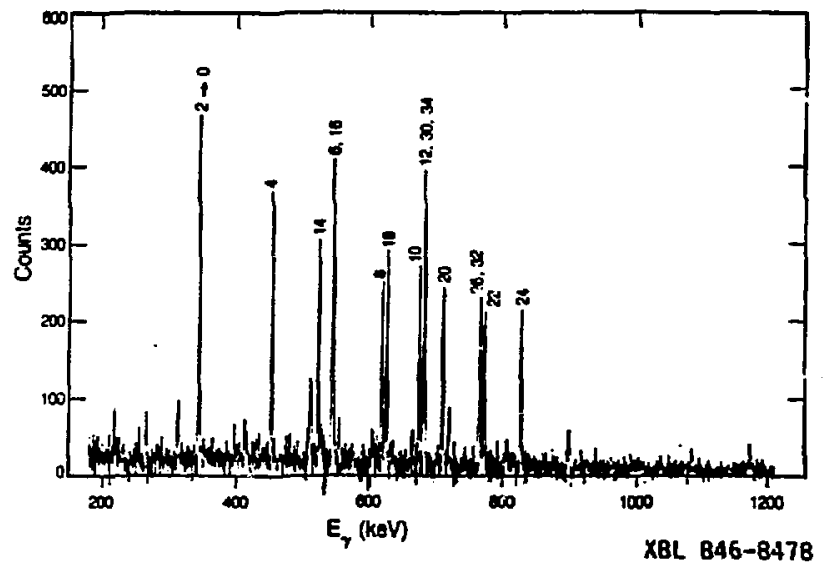

FIGURE 8 Spectrum of $156 \mathrm{Er}$ in coincidence with the 884 $\mathrm{KeV}, 28^{+} \rightarrow 26^{+}$transition.

spectrum shown in Fig. 8. The $v i_{13 / 2}$ backbend at spin 12 in shows clearly, as does the discontinuity at spin $26 \mathrm{~h}$, but aiso one at $30 \mathrm{~h}$. This part of this cascade is no longer rotational, but appears to be made of single-particle states. It would be interesting to determine the transition lifetimes here. Note that in this positive-parity yrast band there are two lines that are double and one that is triple. In fact, of the more than 100 lines Frank has assigned to the decay scheme, more than half are double, triple, or higher fold, and there are six transitions at $766 \neq 1 \mathrm{keV}$. A spectrum brought back as the result of a double gate on $344 \mathrm{KeV}$ is shown in Fig. 9. That is, the $2^{+} \rightarrow 0^{+}$transition of the ground band is weakly coincident with another $r$ ray of the same energy, and the result- 


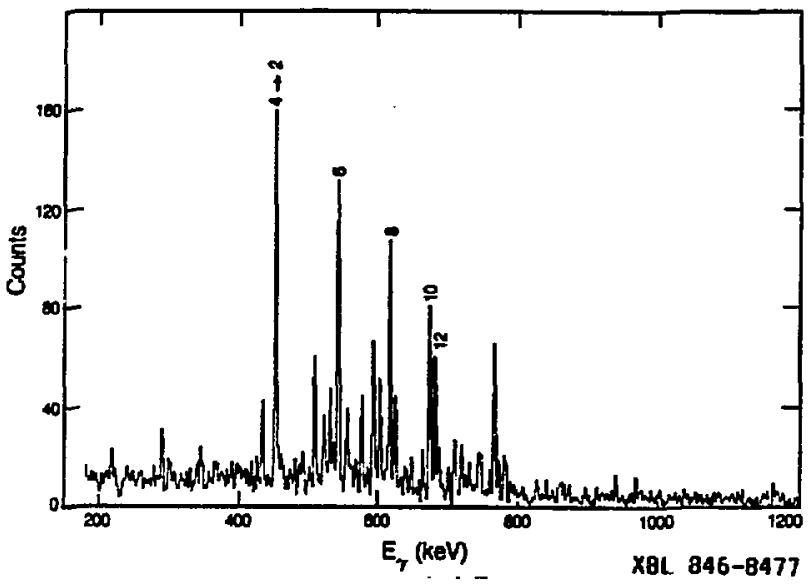

FIGURE 9 Triple coincidence spectrum of $156 \mathrm{Er}$ with a double gate sut on two $344 \mathrm{KeV}$ transitions. The spectrum has been smoothed once using a simple three-channel algorithm.

ing triple-caincidence spectrum is shown. The ground band is observed through the first backbend, and then there are a number of transitions in the energy range of 550-600 KeV which must involve the ather $344 \mathrm{KeV}$ transition, but they have not all been placed in the scheme yet.

The preliminary level scheme for ${ }^{156} \mathrm{Er}$ is given in

Fig. 10. Positive-parity levels above spin $26 \mathrm{~h}$ and negative-parity levels above $-23 \mathrm{~h}$ are mostly new; a few lower levels have been changed from previous work. 4,5 The number of transitions and levels have been doubled over those in earlier schemes. Spin assignments for the new states are tentative and based solely on the angular correlations of the cascade transitions. The new spin and parity assiqn- 
THE BERKELEY HIEH-RESOLUTION BALL

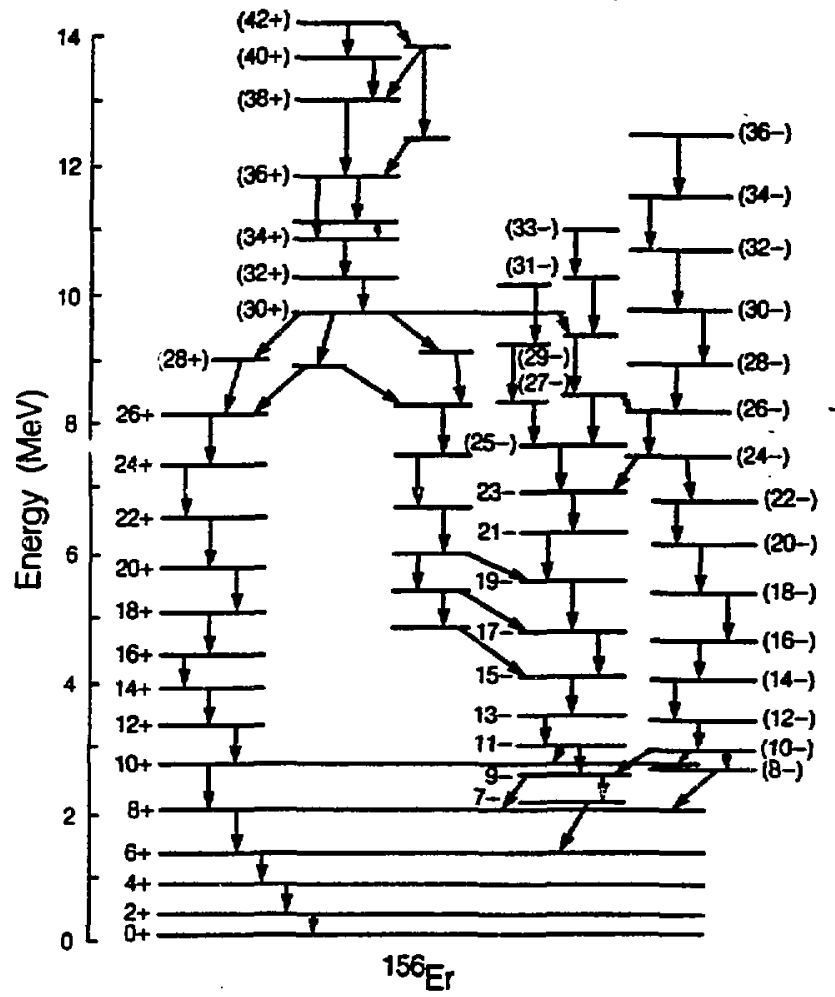

$X 8 L$ 846-8480A

FIGURE 10 Preliminary level scheme for ${ }^{156} \mathrm{Er}$. ments for the right-hand band in the figure are also tentative and will have to be checked by conversionelectron measurements. A plot of energy ys. $I(I+1)$ for the levels is shown in Fig. 11. The lines are drawn only 


\section{R.M. DIAMOND}

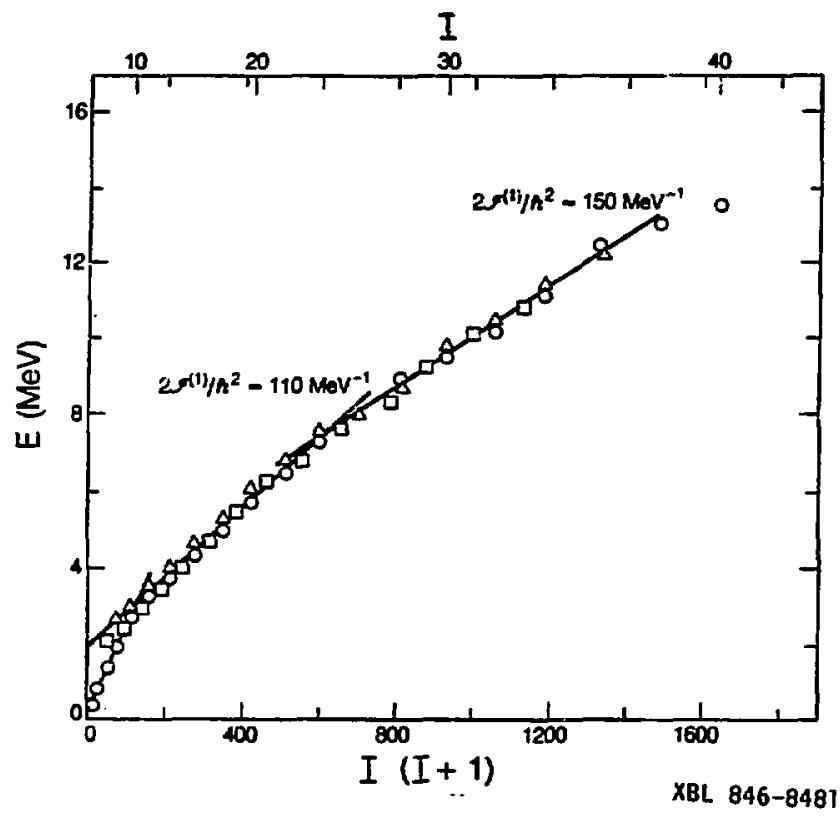

FIGURE 11 Plot of excitation energy $E$ vS. I(I + 1) for the three main bands of $156 \mathrm{Er}$. Corresponding to Fig. 10, they are: the left-most (positiveparity) band, including the non-collective region at the highest spins, circles; the right-nost (negative-parity, even-spin) sequence, triangles; and the center (negativeparity, odd-spin) band, squares.

to guide the eye, but the points do seen to group into three different regions with different slopes. First comes the ground band below spin $10 \mathrm{~h}$ ( 0 quasiparticles) then all bands between spins -12 h and -25 in (2 quasiparticles), and finally all levels above spin -25 h $(4$ or more quasiparti- 
cles). The increasing moment of inertia given by the three slopes is probably the result of decreasing pairing and increasing particle alignment as the number of quasiparticles increases. Although $f(1) / \mathrm{h}^{2}=\mathrm{I} / \mathrm{hu}$ is an integral quantity and so not very sensitive to local structural changes, it is somewhat surprising that all the noints seem to lie so well on the three straight-line segments. The right-hand band in Fig. 10 has been reassigned as a negative-parity band, probably the unfayored even-spin partner of the already assigned negative-parity odd-spin band. Both of these start out with an aligned spin of 7-8 h relative to the ground band and show the "blocked" $\nu i_{13 / 2}$ backbend at hw $\approx 0.35 \mathrm{MeV}$ with an additional alignment of $7-8 \mathrm{~h}$, as expected for the lowest negative-parity bands in this region. Aboye the backbend the odd-spin band splits into two very simflar branches (which was not expected) and the eren-spin band continues, possibly as a still-collective one. This scheme can be compared with Cranked-Shell-Model calculations, ${ }^{6}$ and the agreement is reasonably good, especially for the predicted onset of non-collective or single-particle behavior (oblate shape) taking over from collective rotation (prolate shape) in the neighborhood of spin $30 \mathrm{~h}$. A low-lying calculated state of spin $42 \mathrm{~h}$ is interesting and probably corresponds to the highest spin state observed experimentally and tentatively given that spin. It is possible that the observation of the high-spin positive-parity states even depends upon this state, as it may be the source of the lifetime (>1 picosecond) necessary to hold up the following cascade long enough for the recoiling nucleus to stop first in the lead backing. Otherwise the lines would not be sharp. particularly in the detectors near $90^{\circ}$ to the beam. It 


\section{R.H. DIAMOND}

will be interesting to compare this decay scheme with those of the neighboring nuclef that we are also studying in order to observe the systematics of the changes in nuclear structure and shape in this region of the Periodic Table and see how well they agree with theoretical predictions.

It has become quite clear that the use of Comptonsuppressed Ge detectors is an exciting step forward in nuclear y-ray spectroscopy. The beautiful spectra taken by the Daresbury and Copenhagen groups during the past 1-2 years give eloquent testimony. Their work was done with six large NaI suppressors. The use of bismuth germanate permits much more compact shields, and so allows more $G e$ detectors to be grouped closer to the target or radioactive source than is possible with NaI. As a result, higherorder coincidences $c$ an be obtained which correspond in favorable cases to effectively higher resolution. In addition, adequate statistics can be obtained in a much shorter time. In this talk I have discussed our design of a highresolution Ge array, where it is now in construction, and some first results from a partial array whose spectra do show the superior qualities we had hoped for. In closing I would like to point out that although I have only shown results of discrete r-ray studies, we intend to use the array for continuum work also, as the shielded Ge detector response function is about as good as that of the large NaI detectors usually used in continuum studies. To learn about the properties of the highest spin states we shall have to study their average behavior as given by continuum r-ray measurements; this will require significant improvements in our continuum techniques. But we can look forward to great increases in our knowledge of nuclear structure at high spin and at high temperature from a combination of the 
detailed measurements of discrete y-ray studies and of the average behavior exemplified in continuum work.

Acknowledgements: Maliy people have helped on this project and deserve thanks. I can oniy mention a few: A. Dancosse for the design and construction of the holder, M.K. Lee for the LN filling system, M.K. Lee, D. Landis, and F. Gin for the electronics, and $R$. Belshe for software development. And the project owes most to the thought and care of Frank Stephens.

\section{REFERENCES}

1. TH. LINDBLAD, L.BAGGE, J. BIALKOWSKI, K. FRANSSON and S. LEVEN, On the Energy Resolution of LAr and LXe Ionization Chambers, this Proceedings (Harwood Acadenic Publishers, New York, 1985).

2. P. J. TWIN, P. J. NOLAN, R. ARYAEINEJAD, D. J. G. LOVE, A. H. NELSOH and A. KIRHAH, Huclear Physics, A409, 343e (1983); P. J. TWIN, TESSA: Its Present Status and Future Developments, this Proceedings (Harwood Academic Publishers, Hew York, 1985).

3. R. M. OLAMOND and F. S. STEPHEHS, The High Resolution Ball (proposal) (unpublished, 198 i).

4. A. W. SUNYAR, E. DER MATEOSIAN, O. C. KISTMER, A. JOHNSON, A. H. LUMPKIN and P. THIEBERGER, Physics Letters 62B, 283 (1976).

5. T. BYKSKI, F. A. BECK, C. GEHRINGER, J. C. MERDINGER, Y. SCHUTZ, J. P. VIVIEN, J. DUDEK, W. NAZAREWICZ AND

2. SZ:MANSKI, Physics Letters 102B, 235 (1981).

6. J. DUDEK and W. KAZAREWICZ, private communication (1984). 
This report was done with support from the Department of Enery. Any conchusions or opinions expresed in this report represent soleby those of the author(s) and not necesurily those of The Regents of the University of Californit, the Lawrence Betkeley Leboratory ot the Department of Enery.

Reference to e company or product name does not imply epproval or recommendation of the product by the University of Celifornit or the US. Departenent of Enerty to the exelusion of others that my be suitable. 